\title{
Characterization of Core Optics in Gravitational-Wave Detectors: Case Study of KAGRA Sapphire Mirrors
}

\author{
Eiichi Hirose $\odot,{ }^{1, *}, \dagger$ GariLynn Billingsley $\odot,{ }^{2}$ Liyuan Zhang, ${ }^{2}$ Hiroaki Yamamoto $\odot,{ }^{2}$ Laurent Pinard, ${ }^{3}$ \\ Christoph Michel, ${ }^{3}$ Danièle Forest, ${ }^{3}$ Bill Reichman, ${ }^{4}$ and Mark Gross ${ }^{5, \$}$ \\ ${ }^{1}$ Institute for Cosmic Ray Research, University of Tokyo, Kashiwa, Chiba 277-8582, Japan \\ ${ }^{2}$ LIGO Laboratory, California Institute of Technology, Pasadena, California 91125, USA \\ ${ }^{3}$ Laboratoire des Matériaux Avancés-IP2I CNRS-IN2P3, Villeurbanne 69622, France \\ ${ }^{4}$ Zygo Corporation, Richmond, California 94806 \\ ${ }^{5}$ Commonwealth Scientific and Industrial Research Organisation, West Linfield, New South Wales 2070, Australia
}

(Received 19 March 2020; revised 7 April 2020; accepted 1 June 2020; published 8 July 2020)

\begin{abstract}
We report the characterization of superlow-loss optics used in the second-generation gravitational-wave detectors currently in operation. The sapphire test-mass mirrors in the KAGRA detector are introduced as an example, but the techniques here are common to all detectors. In this work, we discuss mainly the surface topography obtained by interferometric techniques and the optical properties obtained with special setups.
\end{abstract}

DOI: 10.1103/PhysRevApplied.14.014021

\section{INTRODUCTION}

Since the first direct detection of gravitational waves from two approximetely $30 \mathrm{M} \odot$ binary black holes [1], various astronomical events with gravitational waves have been observed. A global network has been formed comprising the two LIGO detectors in the United States [2], the Virgo detector in Europe [3], and the KAGRA detector in Japan [4] for stabler detection and narrower localization of gravitational-wave sources, greatly enhancing the follow-up by electromagnetic observations. All of these gravitational-wave detectors use a kilometer-scale Michelson interferometer that is sensitive to the distortion of spacetime. When gravitational waves arrive as tiny ripples of spacetime, the test masses and end mirrors move along the geodesics to generate a difference in the two arm lengths, defined as the distance between the beam splitter and the end mirrors, resulting in intensity modulation of the laser light at the detection port, which is calibrated into gravitational-wave signals. Since the sensitivity of the detectors is proportional to the arm length, these detectors not only have multikilometer-long arms but also use Fabry-Perot cavities to increase the effective arm length. Since Fabry-Perot cavities with a round-trip loss (RTL; see Sec. II B) of approximately 100 ppm are essential

\footnotetext{
*hirose@inhbar.com

†Present address: inhbar, Inc., Adachi, Tokyo 121-0051, Japan.

$\ddagger$ Present address: Australian National Fabrication Facility NSW Node, School of Electrical Engineering and Telecommunications, Sydney, New South Wales 2052, Australia.
}

for the detection of gravitational waves, the fabrication of ultralow-loss mirrors is required $[5,6]$.

The specifications of these optics are special, and commercial off-the-shelf optics cannot be used. Therefore, state-of-the-art technologies and prototype fabrication are required. Fabrication usually involves the grinding, polishing, and coating of substrates. There are two types of polishing, that are normally used in the final polishing process: conventional pitch polishing and ion-beam figuring (IBF). Similar dielectric multilayered coatings with $\mathrm{Ta}_{2} \mathrm{O}_{5}$ and $\mathrm{SiO}_{2}$ have been applied by ion-beam sputtering to all detectors. The selection of the substrate material is also very important since it determines properties that cannot easily be altered afterwards, such as homogeneity of the refractive index, absorption, mechanical $Q$, thermal conductivity, and stability in heat treatment. In current gravitational-wave-detector networks, either fused silica or sapphire is used as the test-mass material depending on the operating temperature. The LIGO and Virgo detectors are operated at room temperature, while the KAGRA detector is at the cryogenic temperature of $23 \mathrm{~K}$. Sapphire has superior thermal conductivity and mechanical $Q$ at cryogenic temperatures, but there are some issues to address. Previously, we reported some of the issues with reference to a prototype, Pathfinder [7]. However, it is now worth reviewing the real sapphire test-mass optics and providing detailed characterization results. The characterization takes place at several locations, with most of the work being done at the LIGO Laboratory, California Institute of Technology (Caltech), with the Fizeau interferometer and the RTS bench $[8,9]$. Before the characterization, specifications of polishing and coating are evaluated at 
the Zygo Extreme Precision Optics (EPO) facility and Laboratoire des Matériaux Avancés (LMA), respectively. Mechanical loss measurement is performed at the Institute for Cosmic Ray Research (ICRR), University of Tokyo. The thermal stability of sapphire crystal is checked at both the Commonwealth Scientific and Industrial Research Organisation (CSIRO) and LMA.

We hope this article will be useful to both researchers selecting materials for future gravitational-wave detectors and general researchers seeking optics not available on the market. Appendix A summarizes the specifications of the KAGRA test-mass mirrors.

\section{SURFACE TOPOGRAPHY}

We use interferometric techniques to measure the radius of curvature (ROC), surface figure error, inhomogeneity of the refractive index of the substrate, and high-spatialfrequency (HSF) error. Fizeau interferometers are used to measure the figure error and inhomogeneity, while an ADE phase-shift monochromatic phase-measuring microscope (PMM) is used to obtain the HSF error [10]. Figure 1 shows schematic diagrams of the Fizeau interferometer used for surface figure measurement (upper panel) and transmitted-wave-front-error (TWE) measurement (lower panel). For the surface figure-error measurement, the laser light is directly reflected at the front surface. For the TWE measurement, the laser light propagates through the substrate and is reflected at the back surface, from which we can calculate the inhomogeneity and the ROC of the transmitted wave front. Unless otherwise stated, we use the term "inhomogeneity" as the measured TWE originating from the inhomogeneous refractive index and nonuniform thickness after subtracting the Zernike terms $Z_{0,0}, Z_{1,0}, Z_{0,1}$, and $Z_{2,0}$. When a height variation $h(x, y)$ exists between a reference surface and a test surface, a wave-front phase error $\phi(x, y)=4 \pi h(x, y) / \lambda$ will be produced, where $x$ and $y$ are spatial coordinates and $\lambda$ is the wavelength of the laser. By monitoring the phase error at individual pixels of the CCD camera when the position of the reference surface is mechanically modulated, one can determine the surface height $h(x, y)$. This technique is known as phase-shifting interferometry (PSI) and is common to both Fizeau and PMM interferometers. Although we measure the entire surface area (220-mm-diameter aperture, see Table XIV) of the optics to obtain the figure error and ROC, we introduce here the most-important central 140-mm-diameter aperture, which we define as the clear aperture (see Fig. 13).

\section{A. Radius of curvature}

From the height $h(x, y)$ and ROC of the transmission sphere (reference), the ROC of the test piece can be straightforwardly obtained. Table I lists the measured ROC of the coated test-mass mirrors with the root-sum-square of some uncertainties originating from the aperture size,
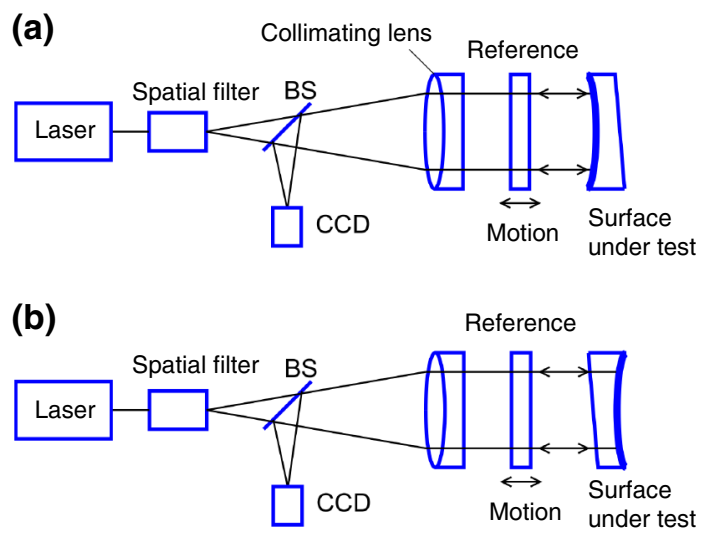

FIG. 1. PSI with two basic measurement settings. The upper panel shows the setting for surface figure and ROC measurement, where the laser light is directly reflected at the front surface. The lower panel shows the setting for TWE measurement, where the laser light propagates through the substrate and is reflected at the back surface, from which we can calculate the inhomogeneity and ROC of the transmitted wave front. BS, beam splitter

the gap between the reference and the test masses, the ROC of the transmission sphere, and the variation of the power term during measurement $[11,12]$. The test-mass mirror near the beam splitter is conventionally called the "input test mass" (ITM), while the test mass far from the beam splitter is called the "end test mass" (ETM). X and $Y$ designate two arms of the Michelson interferometer (see also Fig. 13 in Appendix B). The first and second columns show the ROC and its uncertainty for the high-reflectivity (HR) surface, respectively, while the last column shows the ROC of the transmitted wave front. The values in the first and third columns are reasonably close to the target values, $1900 \pm 9.5 \mathrm{~m}$ and $-1088.3 \mathrm{~m}$, respectively (see also Table XIV).

TABLE I. ROCs within the 140-mm-diameter aperture of the KAGRA test-mass mirrors. The first and second columns show the ROC and its uncertainty for the HR surface, respectively, while the third column shows the ROC of the transmitted wave front. Parentheses indicate that no ROC correction (see Appendix A) is done for the ETMs.

\begin{tabular}{lccc}
\hline \hline Test mass & $\begin{array}{c}\text { ROC of HR } \\
\text { surface }(\mathrm{m})\end{array}$ & $\begin{array}{c}\text { Uncertainty } \\
(\mathrm{m})\end{array}$ & $\begin{array}{c}\text { ROC of } \\
\text { transmitted } \\
\text { wave front } \\
(\mathrm{m})\end{array}$ \\
\hline ITMX & 1904.54 & \pm 2.08 & -1088.0 \\
ITMY & 1904.51 & \pm 2.05 & -1088.3 \\
ETMX & 1907.83 & \pm 2.14 & $(-1114.2)$ \\
ETMY & 1905.09 & \pm 2.10 & $(-1114.7)$ \\
\hline \hline
\end{tabular}




\section{B. Figure error}

By subtracting the height of the reference $h^{\prime}(x, y)$ from $h(x, y)$, we obtain the figure error. Table II shows the figure errors for the coated and uncoated test-mass mirrors, and Fig. 2 shows figure-error maps of the coated test masses within the 140-mm-diameter aperture. The increase in figure error is dominated by the spherical aberration, which appears as a ring pattern in Fig. 2. The target figure error is below $0.5 \mathrm{~nm}$ in the aperture, so we clearly underestimate the increase due to the coating process. The increase in the ETMs being greater than that of the ITMs is because the coating thickness of the ETMs is roughly twice that of the ITMs and also because the figure error of the uncoated ITMs is smaller. Using the figure maps, we estimate the RTL (i.e., the power loss per round trip inside the cavity) of the cavities using STATIONARY INTERFEROMETER SIMULATION [13]. The RTL is found to be approximately $10 \mathrm{ppm}$ for both the $\mathrm{X}$ arm and the $\mathrm{Y}$ arm, which is much smaller than the initially anticipated loss budget for the figure error of $60 \mathrm{ppm}$. The loss budget including the other sources is addressed in Sec. V.

\section{High spatial frequency}

We normally measure the HSF error of the uncoated surface only before coating, but the HR surface of the coated ITMX is investigated this time. The aperture and pixel size are $4.9 \times 4.9 \mathrm{~mm}^{2}$ and $4.74 \times 10^{-6} \mathrm{~m}$ for PMM with $2.5 \times$ objective, while they are $0.2 \times 0.2 \mathrm{~mm}^{2}$ and $2.38 \times 10^{-7}$ $\mathrm{m}$ for PMM with $50 \times$ objective, respectively. We pick nine locations inside the central 140-mm-diameter aperture to perform the measurements; the results are summarized in Table III. Figure 3 shows the HSF maps of the coated ITMX surface measured by PMM $2.5 \times$ and PMM $50 \times$ from one of the nine locations. The rms and peak-to-valley values are 0.14 and $1.55 \mathrm{~nm}$ for PMM $2.5 \times$, while they are 0.11 and $1.11 \mathrm{~nm}$ for PMM 50×, respectively.

\section{Power spectral density}

The power spectral density (PSD) can summarize the figure error and HSF error in a single plot. Figure 4 shows the PSD of the HR surface of the coated ITMX. We distinguish here the figure error, PMM $2.5 \times$, and PMM 50×

TABLE II. Figure errors within the $140-\mathrm{mm}$ aperture of the coated and uncoated KAGRA sapphire test-mass mirrors.

\begin{tabular}{lcc}
\hline \hline Test mass & Coated rms $(\mathrm{nm})$ & Uncoated rms $(\mathrm{nm})$ \\
\hline ITMX & 0.54 & $0.29^{\mathrm{a}}$ \\
ITMY & 0.49 & $0.19^{\mathrm{b}}$ \\
ETMX & 0.92 & 0.35 \\
ETMY & 0.86 & 0.32 \\
\hline \hline
\end{tabular}

${ }^{\text {a }}$ Measured at Zygo EPO.

${ }^{b}$ Measured at Zygo EPO.
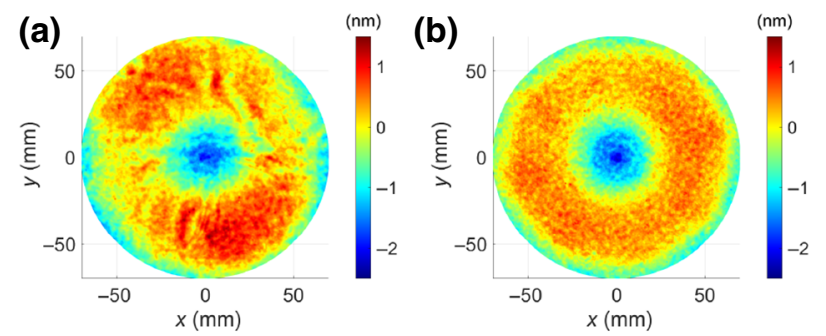

(c)

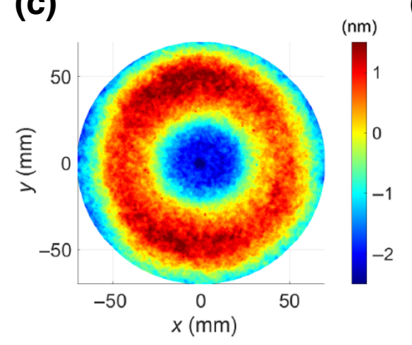

(d)

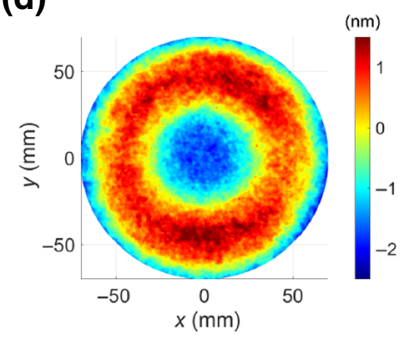

FIG. 2. Figure-error maps of the coated mirrors for the KAGRA test-mass mirrors: (a) ITMX, (b) ITMY. (c) ETMX, and (d) ETMY. The unit of the color bar is nanometers. The spherical aberration is apparent although the rms still remains of subnanometer scale

by their spatial frequency in such a way that the figure error covers below $1 \mathrm{~mm}^{-1}$ (circle 1), PMM $2.5 \times$ covers between 1 and $60 \mathrm{~mm}^{-1}$ (circle 2), and PMM 50× covers between 60 and $1000 \mathrm{~mm}^{-1}$ (circle 3). The blue curve is calculated from the coated surface within the $200-\mathrm{mm}$ diameter aperture, while the solid black line is calculated from the uncoated surface. The two curves agree well except for spatial frequencies below approximately 0.05 $\mathrm{mm}^{-1}$, where the effect of spherical aberration appears. The green and red curves respectively show the PSD of PMM $2.5 \times$ and PMM 50 $\times$ calculated from the maps in Sec. II C, while the dashed and thinner black curves show the PSD for the uncoated equivalents. There is a slight discrepancy at higher spatial frequencies, which indicates some degradation caused by the coating process.

\section{E. Inhomogeneity of the substrate}

The inhomogeneity of the refractive index of a material is not usually categorized as its topography, but we introduce sapphire's inhomogeneity here since the measurement technique is essentially the same as the figure

TABLE III. Average measured rms HSF error and the standard deviation.

\begin{tabular}{lcccc}
\hline \hline Type & $\begin{array}{c}\text { Coated } \\
(\mathrm{nm})\end{array}$ & $\begin{array}{c}\text { Standard } \\
\text { deviation } \\
(\mathrm{nm})\end{array}$ & $\begin{array}{c}\text { Uncoated } \\
(\mathrm{nm})\end{array}$ & $\begin{array}{c}\text { Standard } \\
\text { deviation } \\
(\mathrm{nm})\end{array}$ \\
\hline PMM 2.5 & 0.145 & 0.051 & 0.330 & 0.141 \\
PMM 50× & 0.133 & 0.050 & 0.089 & 0.019 \\
\hline \hline
\end{tabular}



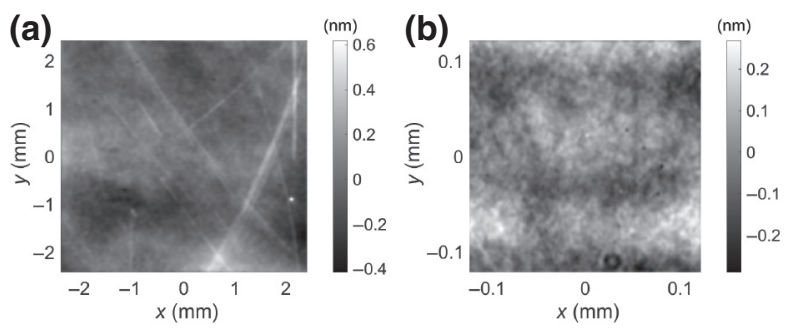

FIG. 3. HSF maps of the coated ITMX HR surface: (a) PMM $2.5 \times$ and (b) PMM 50 $\times$. The rms and peak-to-valley values are 0.14 and $1.55 \mathrm{~nm}$ for PMM 2.5×, while they are 0.11 and 1.11 nm for PMM 50×, respectively.

measurement. Inhomogeneity of the substrate is especially important in the ITMs since wave-front phase distortion while the beam propagates through the substrate degrades the sensitivity [14]. Sapphire is known to have inhomogeneity about 1 order of magnitude greater than that of fused silica. Therefore, to suppress phase distortion, correction of the point-to-point thickness of the back surface by IBF is necessary, which is not needed for fused silica. We specify that the inhomogeneity of the ITMs over a 140-mm-diameter aperture should be less than $6 \mathrm{~nm}$ using STATIONARY INTERFEROMETER SIMULATION [15]. Since a Fizeau interferometer relies on proper alignment of the surface under test with the reference surface, a wedge angle is usually required to avoid reflections between the front and back surfaces, which would destroy the phase information. This situation occurs in the ITMs whose wedge angle is only $0.025^{\circ}$, while the measurement of the ETMs with a wedge angle of $0.05^{\circ}$ is conducted without problems. There are other ways to circumvent this issue. One is, as done for the test masses, to apply an antireflective coating on the back surface of the surface under test. However, the

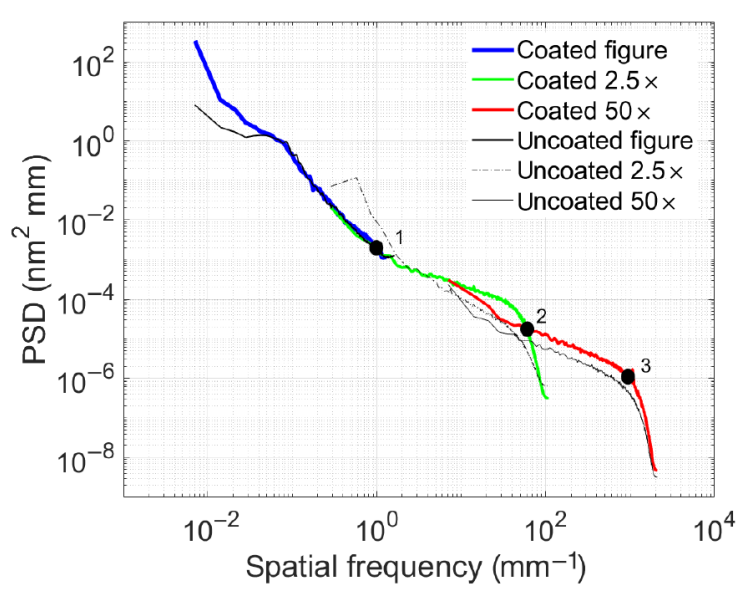

FIG. 4. Power spectral density of ITMX. Both coated and uncoated surfaces are investigated. Some increase due to the coating process can be recognized.
TABLE IV. Fizeau interferometers used in the characterization.

\begin{tabular}{lccc}
\hline \hline Location & Type & Wavelength $(\mathrm{nm})$ & Polarization \\
\hline Caltech & PSI & 1064 & Linear \\
Zygo EPO & PSI and MST & 633 & Circular \\
\hline \hline
\end{tabular}

problem with this approach is that no information about the inhomogeneity is obtained until the coating is complete. Another method is to use a Fizeau interferometer with a wavelength-shifting technology called "MST" (multiple surface test) so that multiple surfaces can be measured simultaneously. Table IV lists the Fizeau interferometers used in the characterization. The ideal system for evaluating the test masses would be a Fizeau interferometer with both PSI and MST technologies, a wavelength of 1064 $\mathrm{nm}$, and linearly polarized light. Table V and Fig. 5 show the inhomogeneity of the coated-test-mass mirrors within the 140-mm-diameter aperture when the direction of linear polarization is parallel to the $x$ axis. In conclusion, the correction fails to satisfy the previously introduced inhomogeneity of $6 \mathrm{~nm}$. Even though inhomogeneity correction is done for the ITMs, the measured value is not so different from that for the ETMs, for which no such correction is performed. Sapphire is a birefringent crystal, but the index of refraction on the plane (the $c$ plane) perpendicular to the $c$ axis would be uniform if the crystal were perfect. We align the $c$ plane with the cylindrical plane to within $\pm 0.1^{\circ}$ using a Bruker D2 CRYSO x-ray-diffraction analyzer since the choice of the crystalline axis should be beneficial for transverse electromagnetic waves. The correction is based on inhomogeneity maps obtained with circularly polarized light instead of the ideal linearly polarized light. If the crystal had been perfect, the choice of polarization would not have been an issue. Namely, the two inhomogeneity maps obtained with circularly polarized light and linearly polarized light would have been identical. The fact that the two maps do not coincide indicates the existence of a local misaligned crystalline structure inside the crystal. Zygo EPO reported the inhomogeneity after IBF correction, which is listed in Table VI. One way to verify these values is to imitate an inhomogeneity map obtained with circularly polarized light by using two maps obtained with

TABLE V. Rms and peak-to-valley (PV) values of inhomogeneity within the 140-mm aperture of sapphire crystals used as the KAGRA test-mass mirrors, measured at Caltech.

\begin{tabular}{lcc}
\hline \hline Test mass & rms $(\mathrm{nm})$ & PV value $(\mathrm{nm})$ \\
\hline ITMX & 25.90 & 177.18 \\
ITMY & 32.72 & 218.19 \\
ETMX & 20.13 & 117.54 \\
ETMY & 94.81 & 392.55 \\
\hline \hline
\end{tabular}




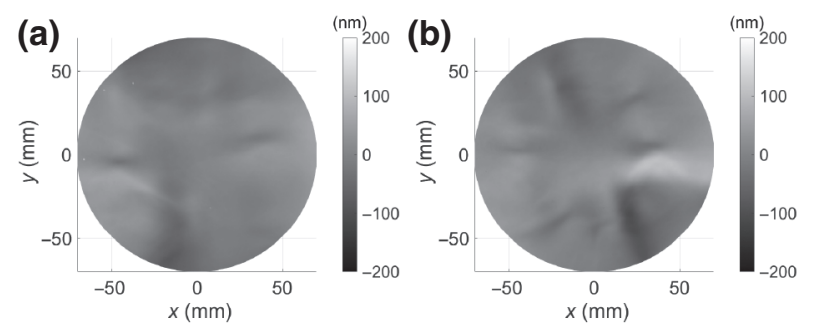

(c)
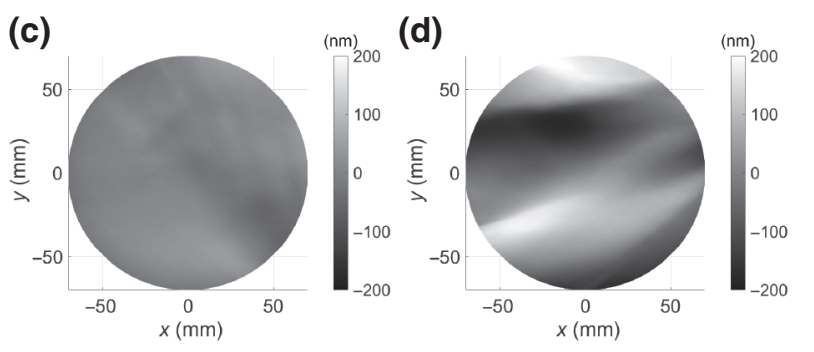

FIG. 5. Measured inhomogeneity maps of the coated-testmass mirrors with linearly polarized light parallel to the $x$ axis: (a) ITMX, (b) ITMY, (c) ETMX, and (d) ETMY.

linearly polarized light. For example, right circularly polarized light $\mathbf{E}$ traveling in the $z$ direction can be expressed by $\mathbf{E}=E_{0}[\hat{\mathbf{i}} \cos (k z-\omega t)+\hat{\mathbf{j}} \sin (k z-\omega t)]$, where $E_{0}, k, \omega$, $\hat{\mathbf{i}}$, and $\hat{\mathbf{j}}$ are the amplitude, propagation number, angular frequency of the electric field $\mathbf{E}$, and unit basis vectors along the $x$ and $y$ directions, respectively. The $90^{\circ}$ phase advance in the $y$ direction can be mimicked by rotating the test piece by $90^{\circ}$. Since the Fizeau PSI guarantees the same amplitude, we can numerically construct data obtained from "circularly polarized light." The constructed maps are similar to those reported by the polisher, and the constructed inhomogeneity values are given in Table VI. The discrepancy may have come from the use of slightly elliptically polarized light by the polisher, who used 633-nm light instead of 1064-nm light, or from some measurement error.

\section{PROPERTIES OF COATING}

The test-mass mirrors currently used in gravitationalwave detectors all use a multilayered dielectric coating formed by alternate layers of fused silica $\left(\mathrm{SiO}_{2}\right)$ and tantala $\left(\mathrm{Ta}_{2} \mathrm{O}_{5}\right)$ made by ion-beam sputtering on the substrate [16]. To characterize optical properties of the coating,

TABLE VI. Reported inhomogeneity within the clear aperture and the numerically constructed rms corresponding to the maps in Fig. 6.

\begin{tabular}{lccc}
\hline \hline Test mass & $\begin{array}{c}\text { Zygo } \\
\text { report } \\
(\mathrm{nm})\end{array}$ & $\begin{array}{c}\text { Constructed } \\
\text { rms (nm) }\end{array}$ & $\begin{array}{c}\text { Peak-to- } \\
\text { valley value } \\
(\mathrm{nm})\end{array}$ \\
\hline ITMX & 3.47 & 4.09 & 41.36 \\
ITMY & 4.07 & 4.61 & 50.32 \\
\hline \hline
\end{tabular}
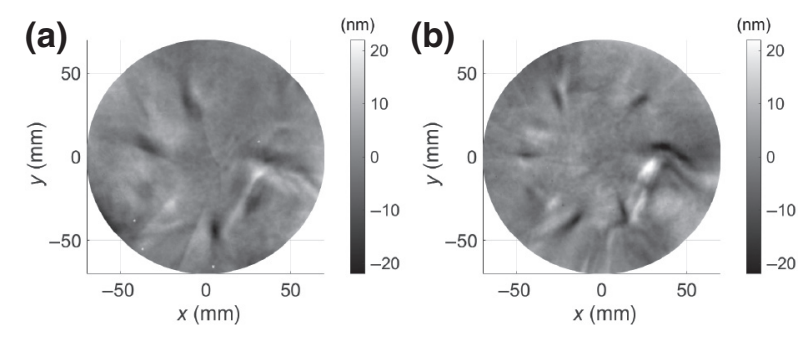

FIG. 6. Inhomogeneity of the sapphire ITMs with circularly polarized light, mimicked by two maps independently measured with linearly polarized light: (a) ITMX and (b) ITMY.

we use the RTS bench at the LIGO Laboratory, Caltech, which is capable of measuring scattering, absorption, transmittance, and reflectance. The setup is described in detail in Ref. [8], and basic schematic diagrams are given in Fig. 7. We use an integrating sphere to measure total integrated scattering (TIS) with a photodetector. This method reveals both the surface roughness, read by the floor level, and point-scattering sources. LMA uses a different technique with a CASI (complete-angle-scatter instrument) scatterometer, which generates somewhat optimistic results. Photothermal common-path interferometory is used to measure absorption. Transmittance and reflectance are straightforward to measure. Except when measuring the absorption of the coating, we scann the central 160-mm-diameter aperture in $1 \mathrm{~mm}$ steps using an $x-y$ stage in the measurement.

\section{A. Scattering of coating}

Table VII and Fig. 8 show the measured TIS of the HR coating of the test-mass mirrors. The most-populated values in the histograms are around a few parts per million, but the many point-scattering sources in the maps increase both the mean and the standard deviation. The minimum values correspond to the roughness of the coated surface. Using the formula $\sigma=\lambda \sqrt{S} /(4 \pi)$ that relates TIS with surface roughness, where $\sigma, \lambda$, and $S$ are the rms surface roughness, the wavelength of the laser, and the measured

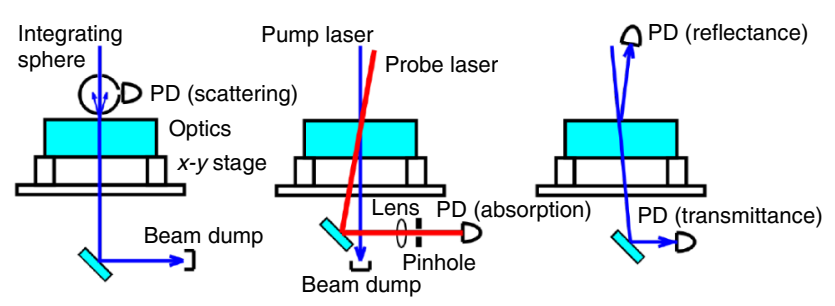

FIG. 7. The RTS bench capable of measuring scattering, absorption, transmittance, and reflectance. The $x-y$ stage allows us to observe these optical properties over a large area. A 160 -mm-diameter aperture is chosen for this study. PD, photodetector. 
TABLE VII. Mean, minimum, and standard deviation of measured TIS of the coating of the test-mass mirrors. The target scattering is smaller than $10 \mathrm{ppm}$.

\begin{tabular}{lccc}
\hline \hline Test mass & Mean $(\mathrm{ppm})$ & $\begin{array}{c}\text { Minimum } \\
(\mathrm{ppm})\end{array}$ & $\begin{array}{c}\text { Standard } \\
\text { deviation } \\
(\mathrm{ppm})\end{array}$ \\
\hline ITMX & 7.10 & 2.843 & 34.06 \\
ITMY & 7.56 & 2.903 & 30.11 \\
ETMX & 11.30 & 2.754 & 49.74 \\
ETMY & 10.95 & 2.699 & 44.34 \\
\hline \hline
\end{tabular}

TIS respectively, $\sigma$ becomes at most about $0.14 \mathrm{~nm}$. This is consistent with results separately obtained by PMM introduced in Sec. II C. Since we carefully clean the surface
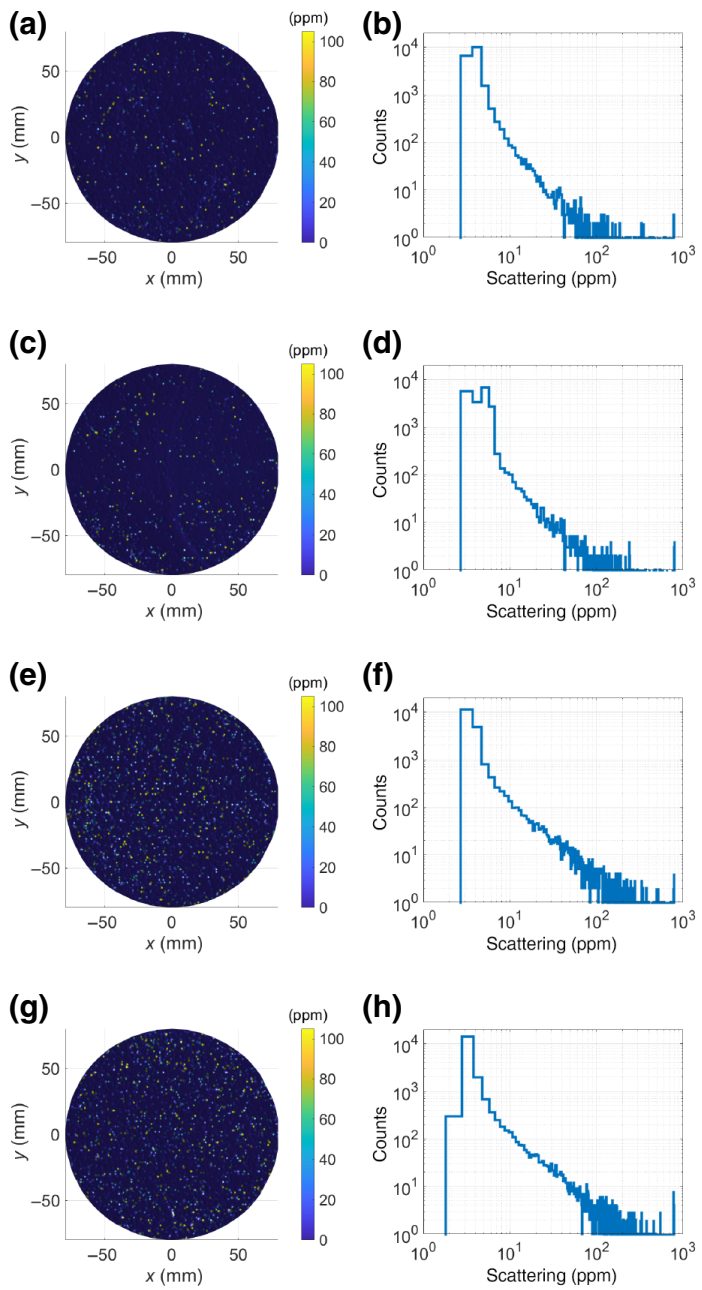

FIG. 8. Measured scattering of the KAGRA test-mass mirrors: (a) ITMX map, (b) ITMX histogram, (c) ITMY map, (d) ITMY histogram, (e) ETMX map, (f) ETMX histogram, (g) ETMY map, and (h) ETMY histogram. Point-scattering sources are distributed over the scanned 160-mm-diameter aperture. The floor levels are almost identical in the mirrors, while the number of point-scattering sources is greater in the ETMs, resulting in larger mean values.
TABLE VIII. Absorption of the coating of the sapphire mirrors used in the KAGRA test-mass mirrors obtained by scanning. The numbers correspond to lines outside the central 120-mm aperture. The mean and standard deviation (in parentheses) are given.

\begin{tabular}{lcccc}
\hline \hline Test mass & $1(\mathrm{ppm})$ & $2(\mathrm{ppm})$ & $3(\mathrm{ppm})$ & $4(\mathrm{ppm})$ \\
\hline ITMX HR & $0.19(0.07)$ & $0.13(0.05)$ & $0.16(0.06)$ & $0.17(0.07)$ \\
ITMX AR & $0.65(0.06)$ & $0.65(0.10)$ & $0.71(0.08)$ & $0.54(0.10)$ \\
ITMY HR & $0.19(0.08)$ & $0.22(0.09)$ & $0.24(0.10)$ & $0.14(0.06)$ \\
ITMY AR & $0.54(0.06)$ & $0.37(0.07)$ & $0.29(0.08)$ & $0.50(0.11)$ \\
ETMX HR & $0.26(0.17)$ & $0.22(0.10)$ & $0.28(0.10)$ & $0.48(0.20)$ \\
ETMY HR & $0.47(0.18)$ & $0.38(0.13)$ & $0.29(0.10)$ & $0.55(0.22)$ \\
\hline
\end{tabular}

before each measurement, it is unlikely that dust particles affected the measurement. The fact that the floor level is the same for the ITMs and ETMs while scattering is greater in the ETMs indicates that the point-scattering sources are inside the coating layers. The origin of the scattering source is currently under investigation [17]. In addition to 1-mm-step scans, $0.3-\mathrm{mm}$-step scans are performed for the central 48-mm-diameter aperture, yielding very similar results.

\section{B. Absorption of coating}

The power of the pump laser in the photothermalcommon-path-interferometory technique is usually several watts, which provides a sufficient optical path difference due to the absorption of the material. Since the power density at the measurement surface is either close to or beyond the damage threshold of the coating [18], we normally perform line scans of $20-\mathrm{mm}$ length outside the central 120 -mm-diameter aperture to minimize the risk of damaging the coating. Table VIII shows the absorption of the coating of the sapphire mirrors. The numbers in the first column correspond to four lines separated from each other by $90^{\circ}$ that run radially from $r=60 \mathrm{~mm}$ to $r=80 \mathrm{~mm}$. As well as the HR surface of the mirrors, we measure the antireflective (AR) surface of the ITMs to estimate their thermal properties, as described later.

\section{Transmittance of coating}

Table IX and Fig. 9 show the measured transmittance of the HR coating of the test-mass mirrors over the central

TABLE IX. Measured transmittance of the coating of the sapphire mirrors used in the KAGRA test-mass mirrors.

\begin{tabular}{lcc}
\hline \hline Test mass & Mean & Standard deviation \\
\hline ITMX & $4.44 \times 10^{-3}$ & $1.76 \times 10^{-5}$ \\
ITMY & $4.79 \times 10^{-3}$ & $2.17 \times 10^{-5}$ \\
ETMX & $6.80 \times 10^{-6}$ & $3.87 \times 10^{-7}$ \\
ETMY & $6.92 \times 10^{-6}$ & $5.16 \times 10^{-6}$ \\
\hline \hline
\end{tabular}


(a)

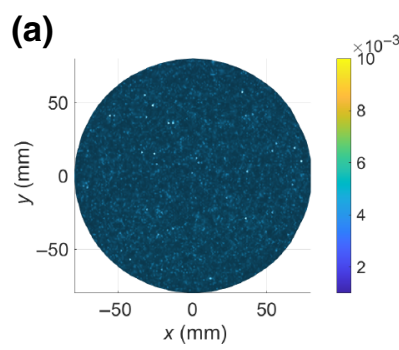

(c)

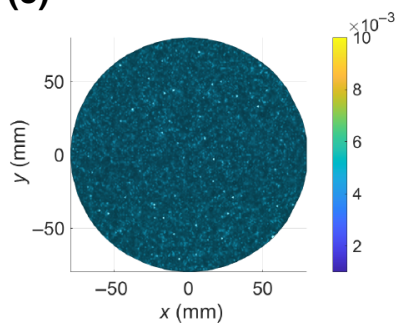

(e)

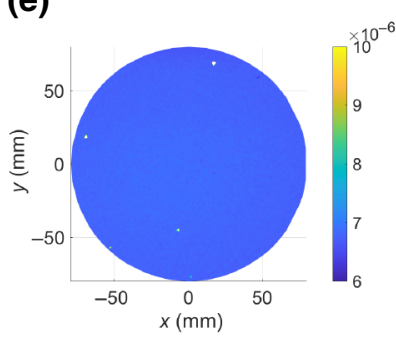

(g)

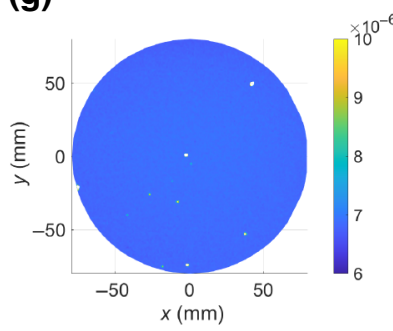

(b)

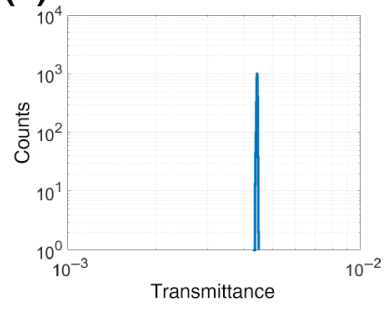

(d)

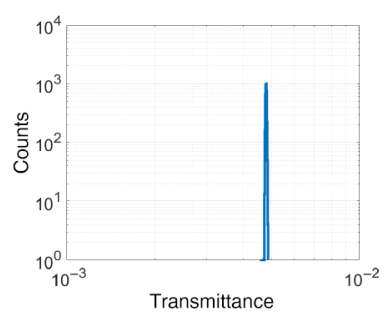

(f)

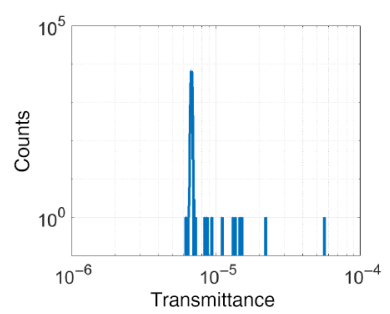

(h)

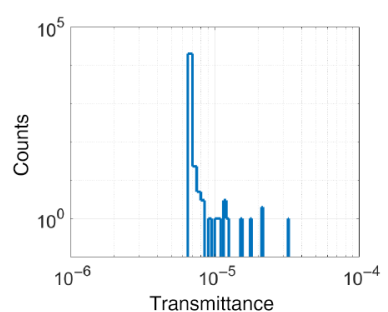

FIG. 9. Transmittance of the KAGRA test-mass mirrors: (a) ITMX map, (b) ITMX histogram, (c) ITMY map, (d) ITMY histogram, (e) ETMX map, (f) ETMX histogram, (g) ETMY map, and (h) ETMY histogram. Several outliers appear, especially for the ETMs, which are recognized in both the maps and the histograms.

$160 \mathrm{~mm}$ aperture. The requirements of the transmittance $T$ of $0.004<T<0.005$ for the ITMs and $5 \times 10^{-6}<T<$ $10 \times 10^{-6}$ for the ETMs are satisfied, as shown. However, the transmission asymmetry, defined by $2\left|T_{1}-T_{2}\right| /\left(T_{1}+\right.$ $T_{2}$ ), where $T_{1}$ and $T_{2}$ are the transmittances of ITMX and ITMY, respectively, is 0.076 , which is greater than the required value of 0.01 . This occurs because we do not coat the two ITMs simultaneously. Instead, we coat them separately owing to a constraint in the project's schedule. The transmittance over the aperture is reasonably uniform for all the mirrors, but there are several points where

TABLE X. Reflectance of the AR coating of the sapphire ITM mirrors in the KAGRA detector, corresponding to Fig. 10.

\begin{tabular}{lcc}
\hline \hline Test Mass & Mean & Standard deviation \\
\hline ITMX AR & $1.857 \times 10^{-4}$ & $5.281 \times 10^{-5}$ \\
ITMY AR & $1.217 \times 10^{-4}$ & $7.607 \times 10^{-5}$ \\
\hline \hline
\end{tabular}

the transmittance greatly exceeds the mean values in the ETMs.

\section{Reflectance of coating}

Table $\mathrm{X}$ and Fig. 10 show the measured reflectance $R$ of the AR coating of the ITM mirrors over the central $160-\mathrm{mm}$ aperture. The measured results satisfy the requirement $1 \times 10^{-4}<R<3 \times 10^{-4}$, although there is some monotonic change in one direction for both mirrors. Also, there are several points in ITMY exhibiting very high reflectance of more than 1 order of magnitude larger than the mean value. Reflectance of the AR coating of the ETMs is not measured by RTS but is measured at LMA, and the measured values are given in Table XIV.

\section{E. Mechanical loss}

Figure 11 shows the measured mechanical loss of the coating on a sapphire disk that is simultaneously coated and annealed with the KAGRA ETM mirrors. The disk is $100 \mathrm{~mm}$ in diameter and $0.5 \mathrm{~mm}$ thick, and we conduct a ring-down measurement to obtain the $Q$ value while changing the temperature of the disk, as described in Ref. [19]. We perform measurements at three different eigenfrequencies, 570, 1338, and $3777 \mathrm{~Hz}$, which correspond to (a)

(c)

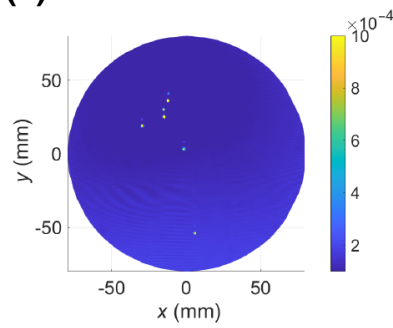

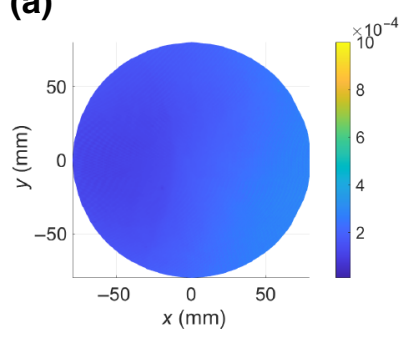

(b)

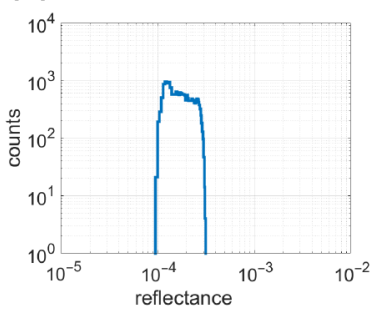

(d)

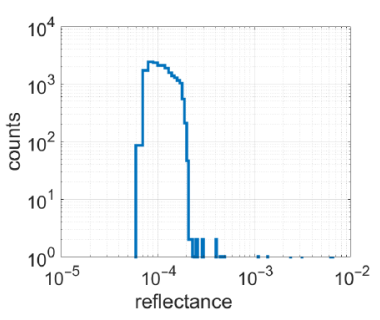

FIG. 10. Measured coating reflectance maps and histograms of the AR coating of the KAGRA input test masses: (a) ITMX map, (b) ITMX histogram, (c) ITMY map, and (d) ITMY histogram. 


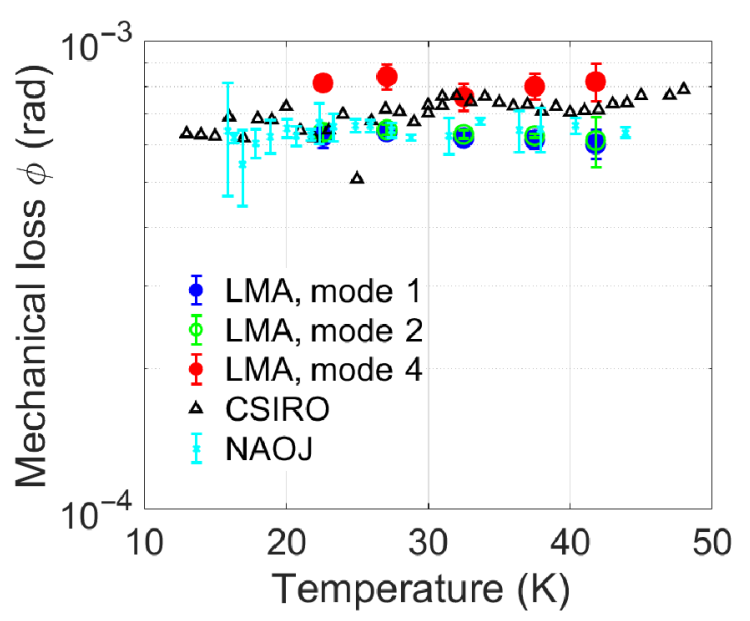

FIG. 11. Measured mechanical loss of the HR coating of the KAGRA ETM mirror. We use a 100-mm-diameter disk simultaneously coated with the test-mass mirrors. The loss is similar to the previously reported result [19] and insensitive to temperature in the range investigated.

modes 1, 2, and 4, respectively, in Fig. 11. For comparison, previously measured results are also plotted. "NAOJ" is the mechanical loss of the disk with a 32-layer coating in Ref. [19] whose mode type corresponds to mode 1, and "CSIRO" is the loss of a disk with a 42-layer coating for mode 1 . The three disks are coated with tantala and silica, but their physical properties probably differ from each other. We use a Young's modulus of $117 \mathrm{GPa}$ for tantala and $70 \mathrm{GPa}$ for silica to calculate the loss [20]. As previously reported, there is no significant temperature dependence of the mechanical loss in the temperature range investigated. "LMA" and "CSIRO" exhibit similar mechanical loss. It is unknown why mode 4 exhibits higher loss.

\section{SUBSTRATE PROPERTIES}

Sometimes materials have properties that are not guaranteed by their manufacturers. In that case, users must confirm that the properties satisfy their needs. For sapphire, homogeneity at $1064 \mathrm{~nm}$, absorption at $1064 \mathrm{~nm}$, and thermal stability to a specific heat treatment are such examples of properties that must be confirmed. The importance of homogeneity was discussed in Sec. II E, so here we discuss the other properties.

\section{A. Thermal stability}

In the final coating process, annealing occurs, which sometimes causes significant deformation of substrate. Thus, it is essential to confirm that the planned annealing results in negligible deformation. We investigate the deformation at two locations: LMA and CSIRO. A sapphire bulk of identical size to the test mass is annealed and the Zernike terms inside the central 140-mm-diameter aperture
TABLE XI. Thermal stability of the sapphire substrate obtained at LMA with a specially prepared substrate. No significant deformation is observed after annealing as a postcoating process.

\begin{tabular}{lcc}
\hline \hline Zernike term & Before annealing $(\mathrm{nm})$ & After annealing $(\mathrm{nm})$ \\
\hline$Z_{2,0}$ & -13.19 & -13.59 \\
$Z_{2,2}$ & 0.40 & 1.08 \\
$Z_{2,-2}$ & -1.73 & -2.12 \\
$Z_{3,1}$ & -0.20 & 0.26 \\
$Z_{3,-1}$ & 0.39 & 0.48 \\
$Z_{4,0}$ & 0.59 & 0.43 \\
\hline \hline
\end{tabular}

before annealing are compared with those after annealing. Table XI shows the results. No significant changes in Zernike terms that may be an issue in controlling surface topography are observed. Using a slightly smaller sapphire crystal of $200-\mathrm{mm}$ diameter used in Pathfinder, a similar test is conducted at CSIRO and no excessive changes in the Zernike terms are observed. These crystals were grown by GT Crystal Systems, who provided the ETM substrates. The ITM substrates were grown by Shinkosha, and they also did not significantly deform, as determined through careful observation of the surface topography. We conclude that sapphire is reasonably stable in terms of deformation on postcoating annealing.

\section{B. Absorption of substrate}

The absorption of high-quality fused silica is about $0.2 \mathrm{ppm} / \mathrm{cm}[21,22]$, while that of sapphire is at least 100 times larger. Material catalogs normally do not specify the absorption at a specific wavelength (1064 nm in our case). However, we must find materials that satisfy a stringent requirement once we try to cool them with a very-high-power injection. The KAGRA Fabry-Perot cavity was designed to have a circulating power of $400 \mathrm{~kW}$. Absorption of the sapphire crystal of as low as $50 \mathrm{ppm} / \mathrm{cm}$ is required for the input test masses [7,23], while the end test masses have a more moderate requirement. It turns out that the requirement is not easy to satisfy for the test-mass size of $220-\mathrm{mm}$ diameter and $150-\mathrm{mm}$ thickness. By investigating several crystals that failed to meet the requirement, we ended up developing "low-absorption" crystals with Shinkosha. Table XII and Fig. 12 show the measured absorption of the crystals used in the test-mass mirrors. The substrates of the ITMs were grown by Shinkosha, while those of the ETMs were grown by GT Crystals Systems. $\mathrm{S} 1, \mathrm{~S} 2$, and S3 are the names of planes parallel to the mirror surface. $\mathrm{S} 1$ and $\mathrm{S} 2$ are about $10 \mathrm{~mm}$ below the surface, while $\mathrm{S} 3$ is located between them. We finally succeeded in developing two crystals that could be used for the ITMs. 
(a)

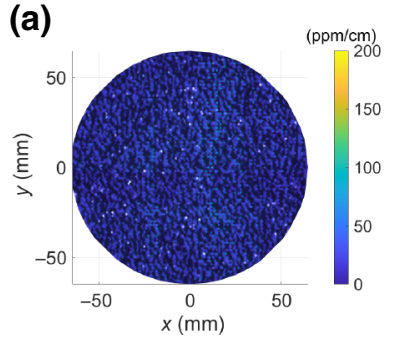

(c)

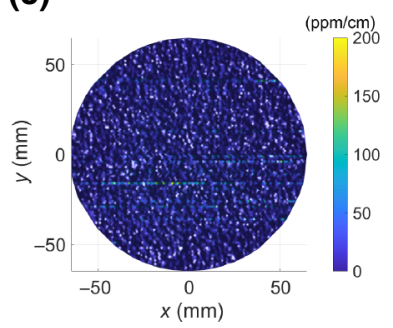

(e)

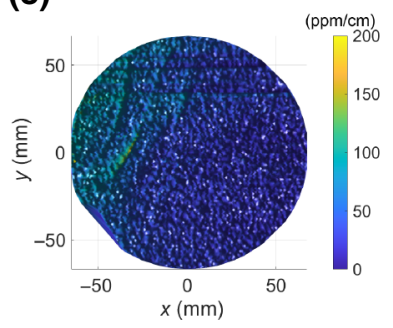

(g)

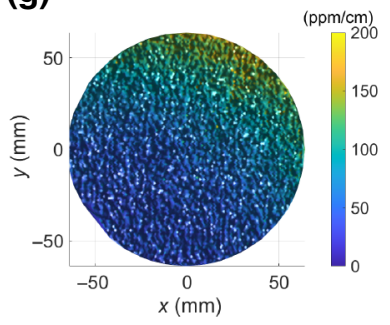

(b)

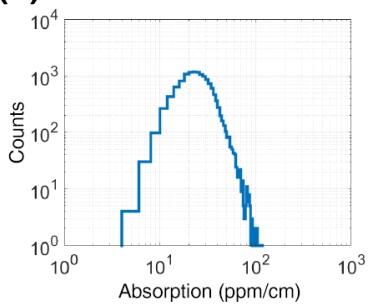

(d)

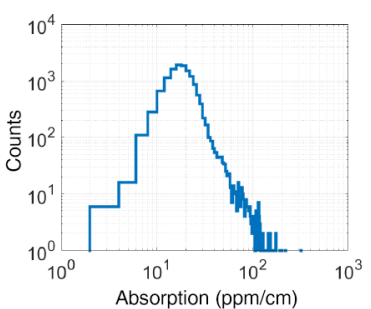

(f)

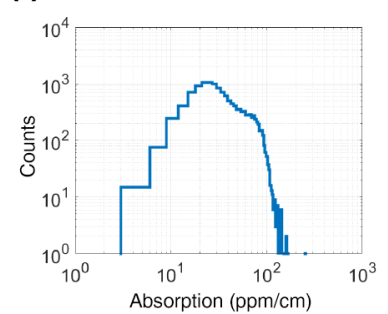

(h)

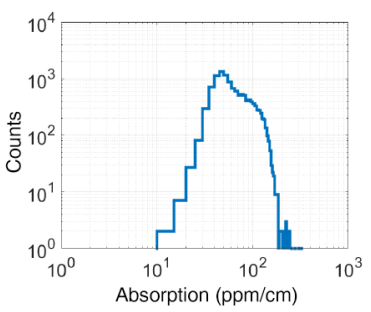

FIG. 12. Measured absorption of sapphire crystals for the testmass mirrors: (a) ITMX map, (b) ITMX histogram, (c) ITMY map, (d) ITMY histogram, (e) ETMX map, (f) ETMX histogram, (g) ETMY map, and (h) ETMY histogram. The unit of the color bar is parts per million per centimeter.

\section{CRYOGENIC FABRY-PEROT CAVITY}

The budget of the RTL of the Fabry-Perot cavity in the KAGRA detector was previously estimated with

TABLE XII. Measured absorption of sapphire crystals used in the test-mass mirrors. The mean and standard deviation (in prantheses) are given.

\begin{tabular}{lccc}
\hline \hline Test mass & $\mathrm{S} 1(\mathrm{ppm} / \mathrm{cm})$ & $\mathrm{S} 2(\mathrm{ppm} / \mathrm{cm})$ & $\mathrm{S} 3(\mathrm{ppm} / \mathrm{cm})$ \\
\hline ITMX & $27.1(11.0)$ & $31.5(10.5)$ & $24.8(10.5)$ \\
ITMY & $21.2(12.3)$ & $22.8(18.2)$ & $33.4(23.4)$ \\
ETMX & $41.2(23.1)$ & $64.3(23.3)$ & $59.9(19.8)$ \\
ETMY & $72.1(31.1)$ & $87.3(38.2)$ & $93.4(36.5)$ \\
\hline \hline
\end{tabular}

TABLE XIII. Loss budget of the KAGRA Fabry-Perot cavities. The total loss is about $50 \mathrm{ppm}$, and there is a sufficient margin to the required $100 \mathrm{ppm}$. Contributions from ITM and ETM are given in parentheses.

\begin{tabular}{lccc}
\hline \hline & Target $(\mathrm{ppm})$ & $\mathrm{X}$ arm $(\mathrm{ppm})$ & $\mathrm{Y}$ arm $(\mathrm{ppm})$ \\
\hline Figure error & $60(30+30)$ & 10 & 10 \\
Scattering & $18(9+9)$ & $18(7+11)$ & $19(8+11)$ \\
Absorption & $2(1+1)$ & $1(0.2+0.5)$ & $1(0.3+0.9)$ \\
ETM transmission & 7 & 6.8 & 6.9 \\
Roughness & $10(5+5)$ & $10(5+5)$ & $10(5+5)$ \\
Defect & $2(1+1)$ & $2(1+1)$ & $2(1+1)$ \\
Diffraction & $1(0.5+0.5)$ & $1(0.5+0.5)$ & $1(0.5+0.5)$ \\
Total & 100 & 49 & 50 \\
\hline \hline
\end{tabular}

Pathfinder [7]. At that time, we did not have real figure maps of the test-mass mirrors or detailed coating properties. Now, with the measured results presented so far, the updated loss budget is given in Table XIII. The total loss is estimated as $50 \mathrm{ppm}$, and there is a sufficient margin to the required $100 \mathrm{ppm}$. As mentioned earlier, the integrating sphere collects both point scattering and scattering due to surface roughness, both of which are listed as scattering. We previously had an item roughness, the loss due to surface roughness alone. We have kept the term for safety even though it means we double-count the contribution. Also, the measured surface quality is better than the listed estimation, but we have retained the original values. The surface quality is measured at Zygo EPO and is given in Appendix A.

The design sensitivity is set assuming that the laser power stored in the Fabry-Perot cavities is $400 \mathrm{~kW}$ [23], although the present operation is performed with a muchmore-moderate power. The required absorption of the sapphire substrate of $50 \mathrm{ppm} / \mathrm{cm}$ is determined so that the test masses can be cooled to $20 \mathrm{~K}$ even when the circulating laser power is $400 \mathrm{~kW}$ inside the cavity. The ITMs are part of the power-recycling cavity, whose circulating power is $800 \mathrm{~W}$ (when both Fabry-Perot cavities store powers of $400 \mathrm{~kW}$ ), and the half-power of $400 \mathrm{~W}$, obtained by splitting with a beam splitter, is continuously input to the ITMs. For the ETMs, the transmitted laser beam is not reflected. Therefore, it is sufficient to examine ITMs to estimate the maximum laser input to the test masses. On the basis of the measured absorption of both the sapphire bulk and the coating of ITMY, the power input from the powerrecycling cavity will be $400 \mathrm{~W} \times(33 \mathrm{ppm} / \mathrm{cm} \times 15 \mathrm{~cm}+$ $0.7 \mathrm{ppm})=0.3 \mathrm{~W}$, while the power input from the Y-arm cavity will be $400 \mathrm{~kW} \times 0.3 \mathrm{ppm}=0.12 \mathrm{~W}$, plus thermal radiation from room temperature of approximately 0.2 $\mathrm{W}$, giving a total of $0.62 \mathrm{~W}$. Since the cooling power of the cryostat is $1 \mathrm{~W}$, it should have been possible to cool the test-mass mirrors to $20 \mathrm{~K}$ without problems. However, the thermal conductivity of the sapphire suspension rods required to remove the total power is lower than expected. 
Therefore, the project set the mirror temperature at $23 \mathrm{~K}$ after an optimization study [24].

\section{IMPACT ON THE DETECTOR}

We mentioned that the current sapphire test-mass mirrors do not satisfy some of the requirements. Among them, the impact of the transmission imbalance and inhomogeneity of the ITM substrate on the detector performance is significant. The transmission imbalance immediately results in imbalance of the arm finesse, which causes commonmode-noise coupling to the gravitational-wave channel. The effect of this asymmetry on the detector sensitivity was numerically studied $[25,26]$. According to the studies, there will be a margin for frequency noise, but intensity noise can limit the design sensitivity. The situation will become worse with inhomogeneity of the ITM substrate. The transfer function from frequency noise to strain sensitivity was independently simulated with the measured inhomogeneity maps, and it was found that the transfer function increases by more than 1 order of magnitude in the observation band [14]. Moreover, the inhomogeneity in the $c$ plane acts as a polarization coupler that converts a linearly polarized incident beam to the opposite polarization state to some extent. A setup was recently built to understand the conversion using different sapphire crystals [27].

\section{CONCLUSION}

We report the characterization of the KAGRA sapphire test-mass mirrors in detail. Although it is slightly more difficult to accomplish ultralow-loss mirrors with sapphire than with fused silica, we show the feasibility of using sapphire. Among the reported failures, transmission imbalance can be solved by coating two ITMs simultaneously, and the requirement for inhomogeneity correction can be satisfied by IBF based on TWE maps obtained with linearly polarized light. However, for future gravitational-wave detectors, research and development of more-homogeneous sapphire crystals in terms of the refractive index in the $c$ plane will be an essential task to achieve higher sensitivity. Although the results here are applicable only to the field of gravitational-wave detectors, we hope that our method for fabricating state-of-the-art large optics will be useful to a wider range of fields.

\section{ACKNOWLEDGMENTS}

This work was partially supported by the JSPS LeadingEdge Research Infrastructure Program, JSPS Grant-inAid for Specially Promoted Research 26000005, and the Joint Research Program of the Institute for Cosmic Ray Research, University of Tokyo. E.H. acknowledges the warm support and accommodation of the LIGO Laboratory, Caltech, and the use of its facilities for characterizing the KAGRA core optics. E.H. also thanks John Tardif and his team members at Coastline Optics for helping the

TABLE XIV. Main specifications of the KAGRA test-mass mirrors. Some requirements and the measured results are given. The values with an asterisk indicate that they do not satisfy the required specifications. The surface defects are inspected before the coating process, so the surface quality of the coated surface is not guaranteed. There is no requirement for measured values in parentheses.

\begin{tabular}{|c|c|c|c|c|c|}
\hline & Requirement & ITMX & ITMY & ETMX & ETMY \\
\hline Diameter (mm) & $220_{-0}^{+0.5}$ & 220.39 & 220.34 & 220.30 & 220.33 \\
\hline Thickness (mm) & $150+0.5 /-0$ & 150.15 & 150.36 & $149.98 *$ & $149.59 *$ \\
\hline $\mathrm{ROC}$ at $140 \mathrm{~mm}(\mathrm{~m})$ & $1900 \pm 9.5$ & 1904.54 & 1904.51 & 1907.83 & 1905.09 \\
\hline Figure at $140 \mathrm{~mm}(\mathrm{~nm})$ & 0.5 & $0.543^{*}$ & 0.487 & $0.919 *$ & $0.864^{*}$ \\
\hline ROC, transmittance at $140 \mathrm{~mm}(\mathrm{~m})$ & -1088.3 & -1088.0 & -1088.3 & $(-1114.2)$ & $(-1114.7)$ \\
\hline Figure, transmittance at $140 \mathrm{~mm}(\mathrm{~nm})$ & 6 & $25.90 *$ & $30.10 *$ & $(35.41)$ & $(51.64)$ \\
\hline \multirow[t]{2}{*}{ Transmittance, HR } & $0.4 \%<T<0.5 \%$ & 0.44 & 0.48 & $\ldots$ & $\ldots$ \\
\hline & $5 \mathrm{ppm}<T<10 \mathrm{ppm}$ & $\ldots$ & & 6.8 & 6.9 \\
\hline Transmission imbalance & 0.01 & \multicolumn{2}{|c|}{$0.076^{*}$} & \multicolumn{2}{|c|}{$\ldots$} \\
\hline Scattering, HR (ppm) & 10 & 7.1 & 7.6 & $11.0^{*}$ & $11.3^{*}$ \\
\hline Absorption, HR (ppm) & 1 & 0.2 & 0.3 & 0.5 & 0.6 \\
\hline Absorption, AR (ppm) & NA & 0.7 & 0.6 & $\ldots$ & $\ldots$ \\
\hline \multirow[t]{2}{*}{ Reflectance, AR (ppm) } & $200 \pm 100$ & 185.7 & 121.7 & $\ldots$ & $\ldots$ \\
\hline & 100 & $\ldots$ & $\ldots$ & $131.2 * \mathrm{a}$ & $45.0^{\mathrm{a}}$ \\
\hline Absorption, substrate $(\mathrm{ppm} / \mathrm{cm})$ & 50 & 32 & 33 & (64) & $(93)$ \\
\hline Defect area at $100 \mathrm{~mm}\left(\mu \mathrm{m}^{2}\right)$ & 2000 & 0 & 0 & 0 & 0 \\
\hline Defect area at $200 \mathrm{~mm}\left(\mu \mathrm{m}^{2}\right)$ & 30000 & 8107 & 142 & 0 & 0 \\
\hline Point defect greater than $2 \mu \mathrm{m}$ count at $100 \mathrm{~mm}$ & 10 & 0 & 0 & 0 & 0 \\
\hline Point defect greater than $2 \mu \mathrm{m}$ count at $200 \mathrm{~mm}$ & 100 & 0 & 0 & 0 & 0 \\
\hline Point defect greater than $2 \mu \mathrm{m}$ density at $200 \mathrm{~mm}\left(\mathrm{~mm}^{-2}\right)$ & 0.25 & 0 & 0 & 0 & 0 \\
\hline
\end{tabular}

${ }^{a}$ Measured at LMA. 


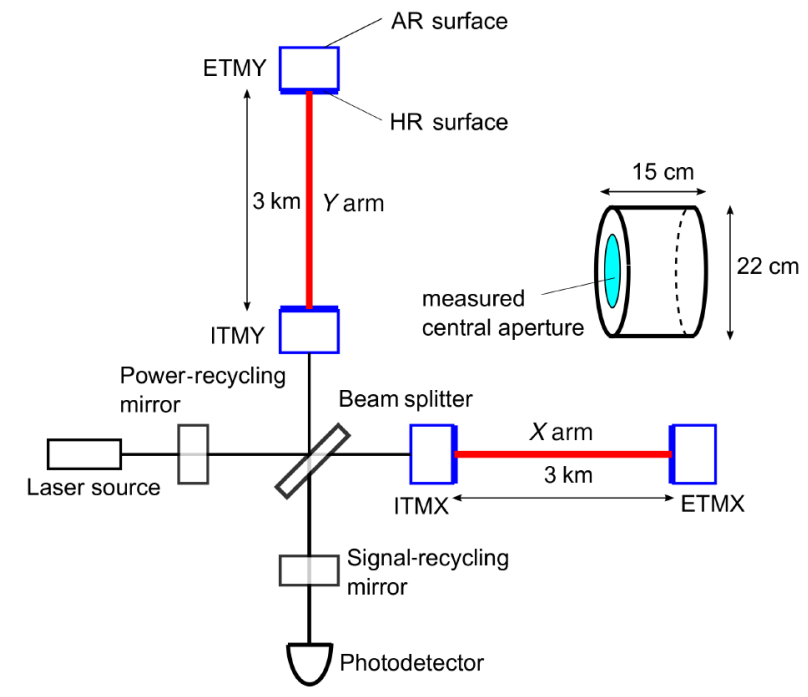

FIG. 13. Simplified diagram (not to scale) of the KAGRA detector. The inset shows the cylindrical shape of the testmass mirror, whose diameter and thickness are 22 and $15 \mathrm{~cm}$, respectively. Characterization occurs inside predefined central apertures as described in the main text.

KAGRA project by preparing various optics, including several sapphire test pieces, in a very quick and accurate manner. E.H. thanks Shinkosha Co. Ltd. for developing low-absorption sapphire crystals that satisfied our requirements. The authors thank Yoichi Aso, Yuta Michimura, and Matteo Leonardi for their useful comments, especially on recent findings in the detector related to the characterization results presented in the article.

\section{APPENDIX A: REQUIREMENTS AND CHARACTERIZATION RESULTS}

Table XIV summarizes the requirements and measured values of the KAGRA sapphire mirrors. The dimension is determined from the largest crystal size available on the market at the time. The ROC of the HR surface, transmittance, and transmission imbalance are determined by the system design [28]. The ROC of the transmitted wave front of the ITM is determined to match the wave front when it is propagating through a perfectly homogeneous substrate, and the ITM is subjected to IBF. The other values are determined to satisfy the RTL requirement [7].

\section{APPENDIX B: SIMPLIFIED DIAGRAM OF KAGRA DETECTOR}

Figure 13 shows a simplified diagram of the KAGRA detector, the Michelson interferometer with two 3-km-long arms made by the test masses. Details of the detector configuration can be found in Refs. [23,28].
[1] B. P. Abbott et al. (LIGO Scientific Collaboration and Virgo Collaboration), Observation of Gravitational Waves from a Binary Black Hole Merger, Phys. Rev. Lett. 116, 061102 (2016).

[2] LIGO, http://www.ligo.caltech.edu/.

[3] VIRGO, http://www.virgo-gw.eu/.

[4] KAGRA, http://gwcenter.icrr.u-tokyo.ac.jp/.

[5] L. Pinard, C. Michel, B. Sassolas, L. Balzarini, J. Degallaix, V. Dolique, R. Flaminio, D. Forest, M. Granata, B. Lagrange, N. Straniero, J. Teillon, and G. Cagnoli, Mirrors used in the LIGO interferometers for first detection of gravitational waves, Appl. Opt. 56, C11 (2017).

[6] J. Degallaix, C. Michel, B. Sassolas, A. Allocca, G. Cagnoli, L. Balzarini, V. Dolique, R. Flaminio, D. Forest, M. Granata, B. Lagrange, N. Straniero, J. Teillon, and L. Pinard, Large and extremely low loss: The unique challenges of gravitational wave mirrors, J. Opt. Soc. Am. A 36, C85 (2019).

[7] E. Hirose, D. Bajuk, G. Billingsley, T. Kajita, B. Kestner, N. Mio, M. Ohashi, B. Reichman, H. Yamamoto, and L. Zhang, Sapphire mirror for the KAGRA gravitational wave detector, Phys. Rev. D 89, 062003 (2014).

[8] L. Zhang, G. Billingsley, and M. Phelps, QA Test Procedure for aLIGO COC, LIGO-E1000863-v2 (2011).

[9] A custom-made setup at the LIGO Laboratory.

[10] P. P. Naulleau, ed., Extreme Ultraviolet (EUV) Lithography $I V$, International Society for Optics and Photonics (SPIE, San Jose, California USA, 2013), Vol. 8679.

[11] E. Hirose, Mechanical loss of the KAGRA ETM coating, JGW-G1910025-v1 (2019).

[12] E. Hirose, Characterization of the coated ITMs, JGWT1809173-v1 (2018).

[13] H. Yamamoto, SIS15, FOG inside, manual, LIGOT1300942-v3 (2018).

[14] K. Somiya, E. Hirose, and Y. Michimura, Influence of nonuniformity in sapphire substrates for a gravitational wave telescope, Phys. Rev. D 100, 082005 (2019).

[15] E. Hirose, Effect of sapphire bulk inhomogeneity on KAGRA IFO, JGW-G1403015-v4 (2014).

[16] M. Granata, E. Saracco, N. Morgado, A. Cajgfinger, G. Cagnoli, J. Degallaix, V. Dolique, D. Forest, J. Franc, C. Michel, L. Pinard, and R. Flaminio, Mechanical loss in state-of-the-art amorphous optical coatings, Phys. Rev. D 93, 012007 (2016).

[17] S. Sayah, B. Sassolas, J. Degallaix, L. Pinard, C. Michel, V. Sordini, and G. Cagnoli, arXiv:1911.02638 (2019).

[18] G. Billingsley, K. Gashwa, M. Phelps, C. Torrie, and L. Zhang, Laser induced damage due to particle contamination, LIGO-G1400209-v2 (2014).

[19] E. Hirose, K. Craig, H. Ishitsuka, I. W. Martin, N. Mio, S. Moriwaki, P. G. Murray, M. Ohashi, S. Rowan, Y. Sakakibara, T. Suzuki, K. Waseda, K. Watanabe, and K. Yamamoto, Mechanical loss of a multilayer tantala/silica coating on a sapphire disk at cryogenic temperatures: Toward the kagra gravitational wave detector, Phys. Rev. D 90, 102004 (2014). 
[20] M. Granata, Personal communication.

[21] R. Dannenberg, G. Billingsley, L. Zhang, and M. Hennig, Bulk absorption measurement of the $\mathrm{CP} 01$ substrate, Heraeus Suprasil 3001, LIGO-T1000456-v1 (2010).

[22] S. Hild, H. Lück, W. Winkler, K. Strain, H. Grote, J. Smith, M. Malec, M. Hewitson, B. Willke, J. Hough, and K. Danzmann, Measurement of a low-absorption sample of OH-reduced fused silica, Appl. Opt. 45, 7269 (2006).

[23] K. Somiya, Detector configuration of KAGRA-the Japanese cryogenic gravitational-wave detector, Classical and Quantum Gravity 29, 124007 (2012).

[24] Y. Michimura, K. Komori, A. Nishizawa, H. Takeda, K. Nagano, Y. Enomoto, K. Hayama, K. Somiya, and M. Ando, Particle swarm optimization of the sensitivity of a cryogenic gravitational wave detector, Phys. Rev. D 97, 122003 (2018).

[25] Y. Enomoto and Y. Michimura, Asymmetry in transmissivity of bKAGRA ITM, JGW-G1707565-v1 (2017).

[26] Y. Enomoto, K. Somiya, and Y. Michimura, On the effect of arm cavity finesse asymmetry to $\mathrm{O} 3$ sensitivity, JGWT1910352-v6 (2019).

[27] S. Zeidler, P. Li, and M. Leonardi, in Symposium on Engineering in Astronomy 2019, Mitaka, Tokyo, 15-17 January (Mitaka, Tokyo, Japan, 2020).

[28] Y. Aso, Y. Michimura, K. Somiya, M. Ando, O. Miyakawa, T. Sekiguchi, D. Tatsumi, H. Yamamoto, and The KAGRA Collaboration, Interferometer design of the KAGRA gravitational wave detector, Phys. Rev. D 88, 043007 (2013). 